\title{
Nonstoichiometric transfer during laser ablation of metal alloys
}

\author{
Canulescu, Stela; Döbeli, Max; Yao, Xiang; Lippert, Thomas; Amoruso, Salvatore; Schou, Jørgen
}

Published in:

Physical Review Materials

Link to article, DOI:

10.1103/PhysRevMaterials.1.073402

Publication date:

2017

Document Version

Publisher's PDF, also known as Version of record

Link back to DTU Orbit

Citation (APA):

Canulescu, S., Döbeli, M., Yao, X., Lippert, T., Amoruso, S., \& Schou, J. (2017). Nonstoichiometric transfer during laser ablation of metal alloys. Physical Review Materials, 1(7), [073402].

https://doi.org/10.1103/PhysRevMaterials.1.073402

\section{General rights}

Copyright and moral rights for the publications made accessible in the public portal are retained by the authors and/or other copyright owners and it is a condition of accessing publications that users recognise and abide by the legal requirements associated with these rights.

- Users may download and print one copy of any publication from the public portal for the purpose of private study or research.

- You may not further distribute the material or use it for any profit-making activity or commercial gain

- You may freely distribute the URL identifying the publication in the public portal

If you believe that this document breaches copyright please contact us providing details, and we will remove access to the work immediately and investigate your claim 


\title{
Nonstoichiometric transfer during laser ablation of metal alloys
}

\author{
Stela Canulescu, ${ }^{1, *}$ Max Döbeli, ${ }^{2}$ Xiang Yao, ${ }^{3}$ Thomas Lippert, ${ }^{3}$ Salvatore Amoruso, ${ }^{4}$ and Jørgen Schou ${ }^{1}$ \\ ${ }^{1}$ Department of Photonics Engineering, Technical University of Denmark, 4000 Roskilde, Denmark \\ ${ }^{2}$ Ion Beam Physics, ETH Zurich, Switzerland \\ ${ }^{3}$ Paul Scherrer Institut, $\mathrm{CH}-5232$ Villigen PSI, Switzerland \\ ${ }^{4}$ Dipartimento di Fisica "Ettore Pancini”, Università di Napoli "Federico II”, Complesso Universitario di Monte S. Angelo, \\ Via Cintia, I-80126 Napoli, Italy
}

(Received 14 June 2017; published 11 December 2017)

\begin{abstract}
Large angular variations in film composition have been found for ablation of a metallic $\mathrm{AuCu}$ alloy $(\mathrm{Au} / \mathrm{Cu}$ ratio $\sim 1$ ) in vacuum and background gases of $\mathrm{Ne}$ and $\mathrm{Xe}$. The $\mathrm{AuCu}$ films grown in vacuum at a laser fluence of $5 \mathrm{Jcm}^{-2}$ exhibit a large loss in the $\mathrm{Cu}$ content, with the $\mathrm{Au} / \mathrm{Cu}$ ratio $\sim 2.4$ at angles close to normal incidence. At this fluence, a distortion of the plume front is observed followed by the appearance of a secondary emission at the substrate, suggesting that resputtering of the film by energetic ions and reflection of ions/atoms at the substrate can lead to a nonstoichiometric transfer in pulsed laser deposition. Further, we have found that depending on the mass of the background gas employed during growth ( $\mathrm{Ne}$ or $\mathrm{Xe}$ ), the ratio of elements in the film can vary significantly over a wide range of angles of deposition. In the presence of the light gas $\mathrm{Ne}$, the degree of nonstoichiometric transfer is gradually reduced with increasing background pressure, resulting in a nearly stoichiometric $\mathrm{AuCu}$ films at a Ne pressure of 2 mbar. The behavior in the heavy gas Xe is more complex, and both theoretical and experimental data indicate that the loss of $\mathrm{Cu}$ in the deposits is caused by the preferential scattering, as well as by backscattering of the light $\mathrm{Cu}$ atoms in the plume upon collisions with the background gas.
\end{abstract}

DOI: 10.1103/PhysRevMaterials.1.073402

\section{INTRODUCTION}

Pulsed laser deposition (PLD) has emerged as a versatile technique that allows the growth of complex structures, including thin oxide films and superlattices [1]. It is generally stated that the ablation process in PLD results in a stoichiometric transfer of material from the target to the substrate, provided that an energy threshold is exceeded. Indeed, when the laser fluence is below a certain threshold, one can expect a strong deviation in the composition of the deposits compared to the target, more predominantly for compounds containing volatile components [2]. For example, a strong dependence on the laser fluence has been observed for the $\mathrm{Cu}_{2} \mathrm{ZnSnS}_{4}$ (CZTS) films deposited by PLD. The CZTS films deposited at a low fluence of $0.2 \mathrm{Jcm}^{-2}$ are $\mathrm{Cu}$ free, and the $\mathrm{Cu}$ content in the films increases with increasing laser fluence [3]. Ohnishi et al. [4] have found that the composition of the PLD-deposited $\mathrm{SrTiO}_{3}$ films can be varied from Sr-rich to Ti-rich with increasing laser energy. Similar findings were reported by Wicklein et al. [5]. A noncongruent material transfer has been reported in PLD for materials containing different atomic masses due to preferential scattering of the light species in the plume, such as $\mathrm{LiMn}_{2} \mathrm{O}_{4}$ [6], $\mathrm{LaAlO}_{3}$ [7], and $\mathrm{SrTiO}_{3}$ [5]. In case of $\mathrm{LiMn}_{2} \mathrm{O}_{4}$, a lithium-rich target was used to compensate for lithium losses in the films [8]. Extensive research has been dedicated to complex perovskite oxides, such as $\mathrm{La}_{0.6} \mathrm{Ca}_{0.4} \mathrm{MnO}_{3}$ (LCMO) or $\mathrm{LaAlO}_{3}$ (LAO) [9], where an angle-dependent composition has been seen [10]. Other microscopic effects, such as self-sputtering and backscattering of the plume species upon arriving at the

*Corresponding author: stec@ fotonik.dtu.dk substrate can contribute to a noncongruent transfer in PLD $[11,12]$.

In the present paper, we report that ablation of a metallic $\mathrm{AuCu}$ target in vacuum and background gases of $\mathrm{Ne}$ and $\mathrm{Xe}$ results in a significant deviation from a stoichiometric transfer in $\mathrm{PLD}$, with the $\mathrm{Au} / \mathrm{Cu}$ ratio in the deposits depending strongly on the laser fluence, mass and pressure of the background gas. The material under study is a completely miscible $\mathrm{AuCu}$ metal alloy with the $\mathrm{Au} / \mathrm{Cu}$ ratio $\sim 1$. The target constituents have similar physical and chemical properties (see Table I), except for their atomic mass. The ablation studies were performed in noble gases, such that chemical effects (oxidation), which can otherwise change the composition of the film, are negligible. In such a particular system, the composition of the films is primarily determined by the mass of the target constituents. We will qualitatively estimate the ablation of the metallic alloy by studying the composition of the deposits over a wide range of angles. For understanding the plume expansion in vacuum, an analysis of the plume dynamics, as a tool to better understand the strong deviation in composition of the deposited films, will be presented.

\section{EXPERIMENTAL}

Thin $\mathrm{AuCu}$ films were deposited at room temperature from ablation of a metallic AuCu target (1:1) on a hemispherical array of Si substrates of radius of $60 \mathrm{~mm}$ (Fig. 1). The AuCu target was fabricated by the arc melting method from high-purity $\mathrm{Au}$ and $\mathrm{Cu}$ granules (purity higher than $99.9 \%$ from Dansk Ædelmetal). The composition of the target was checked by EDX (energy-dispersive x-ray spectroscopy) using a TM3000 instrument. The ablation was done using a frequency-tripled $\mathrm{Nd}$ :YAG laser (wavelength of $355 \mathrm{~nm}$ ) running at a repetition frequency of $10 \mathrm{~Hz}$, with the laser beam directed on the 
TABLE I. Basic properties of elements

\begin{tabular}{lcc}
\hline \hline Element & $\mathrm{Au}$ & $\mathrm{Cu}$ \\
\hline Mass (amu) & 196.96 & 63.54 \\
Cohesive energy (eV/atom) & 3.85 & 3.5 \\
Ionization potential $(\mathrm{eV})$ & 9.22 & 7.72 \\
Thermal diffusivity $(\mathrm{W} / \mathrm{cmK})$ & 3.2 & 4 \\
\hline \hline
\end{tabular}

target at normal incidence. This geometry was chosen in order to achieve a circular beam spot on the target that provides a rotational symmetry for the angle-resolved compositional studies and prevents any flip-over effects [13]. The laser spot size was $5 \mathrm{~mm}^{2}$. The laser beam was only rastered along the vertical direction, such that in the horizontal plane the beam was kept at a fixed position. The $\mathrm{Si}$ substrates were mounted at angles varying from $-90^{\circ}$ to $90^{\circ}$ with respect to normal incidence, as indicated in Fig. 1. The depositions were carried out in vacuum at a laser fluence of 2.5 and $5 \mathrm{Jcm}^{-2}$, base pressure of $\sim 2 \times 10^{-6} \mathrm{mbar}$, Ne background gas $(0.1 \mathrm{mbar}$, 0.5 mbar, and 2 mbar) and Xe background gas (0.01 mbar). The experiments in gas were carried out at a fluence of $5 \mathrm{Jcm}^{-2}$ only. Rutherford backscattering spectrometry (RBS), as interpreted using the RUMP software, was used to measure the composition and thickness of the films. The measurements were taken using a $2 \mathrm{MeV}{ }^{4} \mathrm{He}$ ion beam. For a selection of samples, a higher ion beam energy $\left(5 \mathrm{MeV}^{4} \mathrm{He}\right)$ was used in order to quantify the uniform film including the particulates (droplets). The composition of the films including the droplets showed a similar trend, suggesting that the droplets do not change the overall composition of the deposits.

The plasma generated by ablation of the $\mathrm{AuCu}$ target in vacuum was imaged using a time-gated ICCD camera Andor New i-star, with spectral range from 200-1000 nm and $1024 \times$ 1024 pixels size. More details about the instrumentation are given elsewhere [14]. The images were recorded for all light

\section{Laser beam}

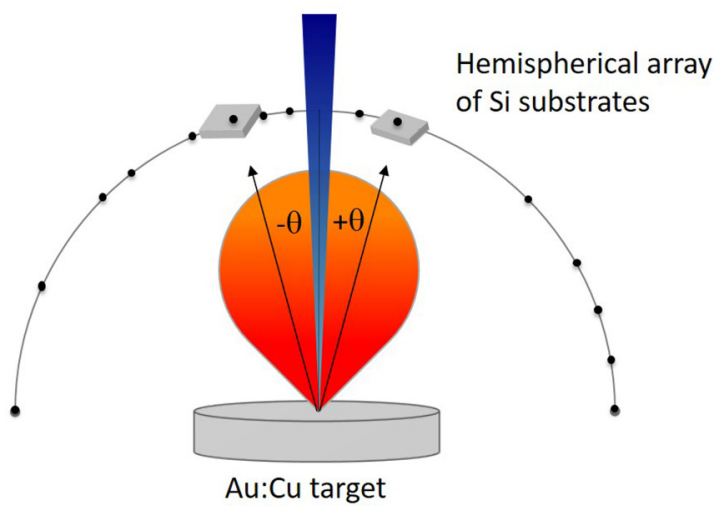

FIG. 1. Schematic of the PLD setup showing the Si substrates placed on a hemispherical array of radius $60 \mathrm{~mm}$. The substrates were placed at $-5^{\circ},-10^{\circ},-15^{\circ},-24^{\circ},-38^{\circ},-45^{\circ},-65^{\circ}$, and $-90^{\circ}$ (left-hand angles) and $8^{\circ}, 15^{\circ}, 45^{\circ}, 60^{\circ}, 70^{\circ}, 80^{\circ}$, and $90^{\circ}$ (right-hand angles), as indicated by the black circles. The laser beam hits the target at normal incidence. For simplicity only two substrates are shown.

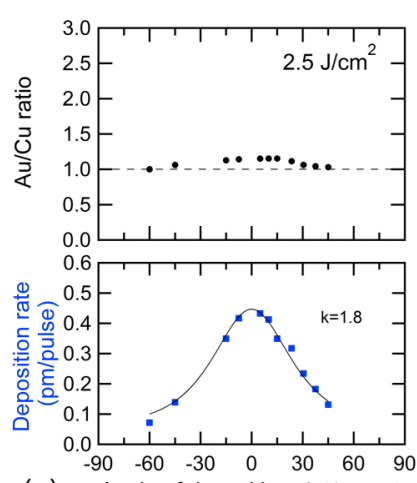

(a) Angle of deposition, $\theta$ (degrees)

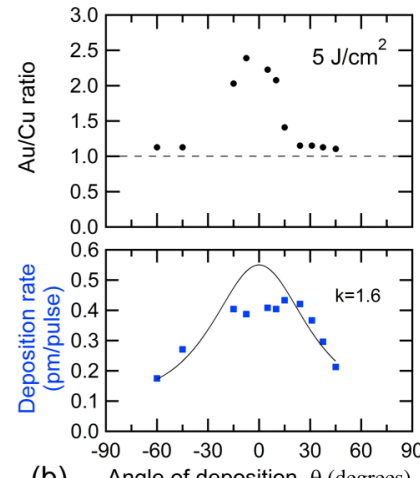

(b) Angle of deposition, $\theta$ (degrees)
FIG. 2. Top plots: Ratio of elements $(\mathrm{Au} / \mathrm{Cu})$ in the films versus angle of deposition $(\theta)$ for ablation in vacuum at a fluence of (a) $2.5 \mathrm{Jcm}^{-2}$ and (b) $5 \mathrm{Jcm}^{-2}$. The dashed lines represent the composition of the target. Bottom plots: The angular distribution of the deposits fitted using Anisimov's model.

passing through a quartz window. For plume imaging and mass spectroscopy analysis of the plume, the laser beam was incident on the target at an angle of $45^{\circ}$, while the laser fluence was set to similar values as the ones used for thin film deposition $\left(2.5\right.$ and $\left.5 \mathrm{Jcm}^{-2}\right)$. The base pressure in the analysis chamber was $\sim 2 \times 10^{-6}$ mbar. The energy spectra of the ions in the plume were measured using an energy analyzer (Hiden Analytical) placed at a distance of $60 \mathrm{~mm}$ from the target.

\section{RESULTS}

The angle-dependent composition profiles of the films deposited in vacuum at a laser fluence of 2.5 and $5 \mathrm{Jcm}^{-2}$ are shown in Figs. 2(a) and 2(b), respectively (top plots). The angle of deposition $(\theta)$ equal to zero corresponds to the normal incidence of the laser beam on the target and the nearest film was deposited at an angle of $5^{\circ}( \pm 1)$. The dashed lines represent the composition of the target, i.e., $\mathrm{Au} / \mathrm{Cu} \sim 1( \pm 0.02)$, as estimated by EDX. At a fluence of $2.5 \mathrm{Jcm}^{-2}$ the deposition is nonstoichiometric, with the $\mathrm{Au} / \mathrm{Cu}$ ratio in the films of $\sim 1.2$. With increasing laser fluence to $5 \mathrm{Jcm}^{-2}$ the degree of nonstoichiometric transfer is significantly enhanced, and the $\mathrm{Au} / \mathrm{Cu}$ ratio in the films peaks at $\sim 2.4$ for the deposits collected at near incidence $\left(\theta \sim 5^{\circ}\right)$. This is equivalent to a loss in the $\mathrm{Cu}$ content of $\sim 70 \%$ as compared to the composition of the target. The dramatic $\mathrm{Cu}$ nonstoichiometry in the films vanishes rapidly at large angles $\left(\theta>30^{\circ}\right)$, suggesting that the mechanisms leading to the nonstoichiometric transfer involve processes whose effectiveness is larger along the plume expansion, as it will be discussed later.

The angular thickness profiles of the deposits, as determined by RBS, are shown in Fig. 2 (bottom plots). Anisimov's model was used to fit the angular distribution of the deposits [15]. According to the model, the angular distribution $F_{A}(\theta)$ of the particle flow onto a hemispherical substrate with an angle $\theta$ relative to the target normal can be expressed as [16]:

$$
F_{A}(\theta) / F_{A}(0)=\left(1+\tan ^{2} \theta\right)^{3 / 2} /\left(1+k^{2} \tan ^{2} \theta\right)^{3 / 2},
$$

where $F_{\mathrm{A}}(0)$ is the value for particles along the target normal at $\theta=0$. The parameter $k$ is the ratio of the cloud front along 

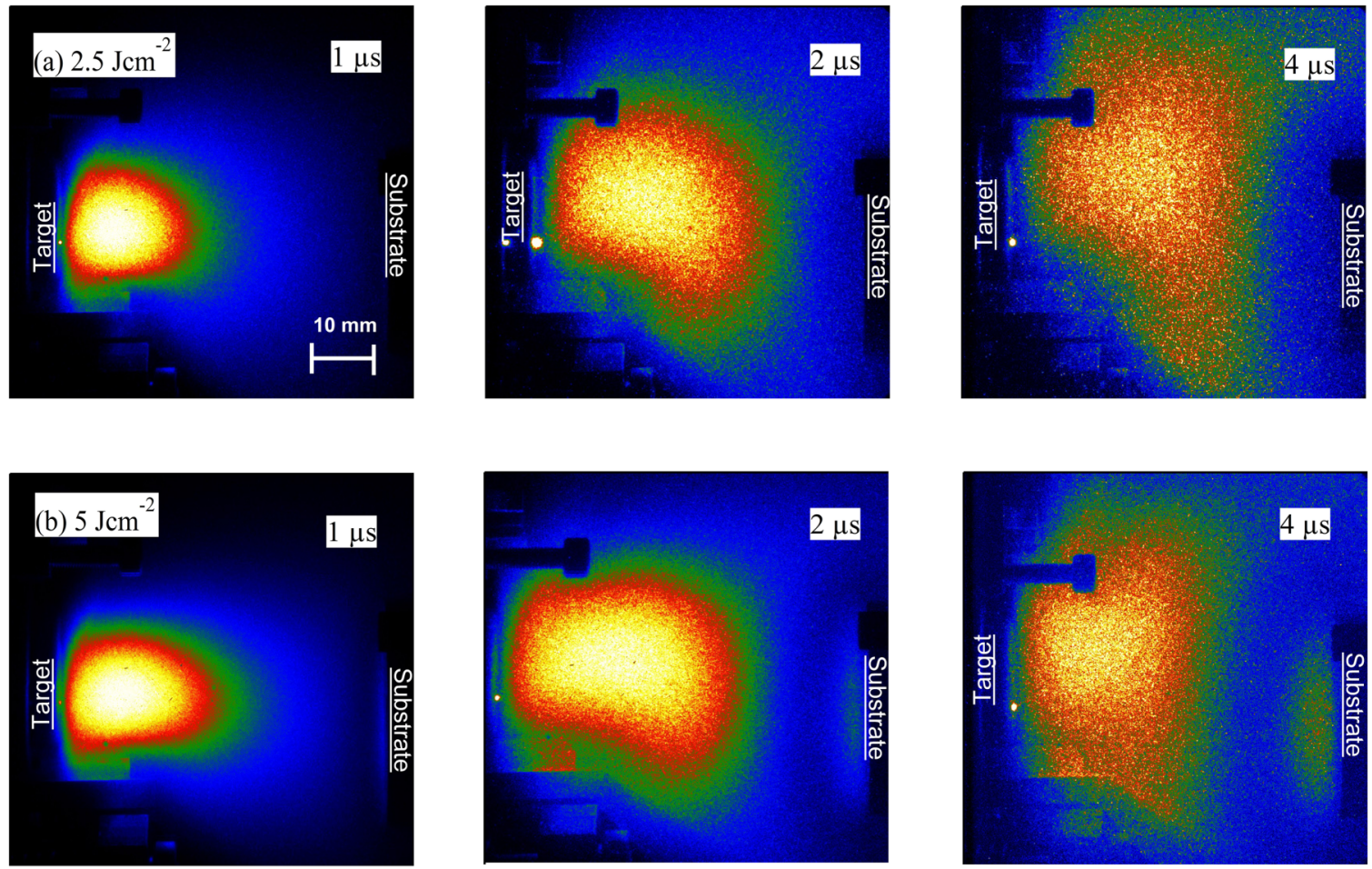

FIG. 3. Time-gated images of the plasma-plume generated by ablation of AuCu in vacuum at a fluence of (a) $2.5 \mathrm{Jcm}^{-2}$ and (b) $5 \mathrm{Jcm}^{-2}$. The images were recorded for a gate width of $1 \mu \mathrm{s}$ and time delay indicated in each image. The target to substrate distance was $60 \mathrm{~mm}$.

the expansion axis $Z\left(Z_{\text {inf }}\right)$ versus the value of the cloud front along the $X$ axis $\left(X_{\text {inf }}\right)$, i.e., $k=Z_{\text {inf }} / X_{\text {inf. }}$. In other works, $k$ defines the angular spread of the flux; the larger the $k$ value the more peaked the plume expansion in the forward direction is. Fitting the angular thickness profile at a fluence of $2.5 \mathrm{Jcm}^{-2}$ yielded $k=1.8$. We note that the deposition rate per pulse does not change significantly with increasing fluence from 2.5 to $5 \mathrm{Jcm}^{-2}$ at low angles, but it rises at the wings. We anticipate that resputtering effects are likely responsible for a flat-top thickness profile at high fluence, which also implies that the thickness profile could not be well described by Anisimov's model [Fig. 2(b), bottom panel].

In order to have a better understanding of the plume dynamics in vacuum, plume imaging and mass spectrometry analyses were performed. A series of time-resolved images of the plasma-plume expansion at 2.5 and $5 \mathrm{Jcm}^{-2}$ is shown in Figs. 3(a) and 3(b), respectively. All images were obtained using a gate width of $1 \mu \mathrm{s}$ and each image is normalized to its brightest intensity pixel for an easy comparison. The positions of the target and substrate holder are indicated in each image. Shortly after the laser pulse, the plume expands freely into vacuum, more dominantly peaked along the normal direction. At a delay time of $2 \mu \mathrm{s}$, the plume front reaches the substrate and the plume emission becomes distorted. At the high fluence of $5 \mathrm{Jcm}^{-2}$ a secondary plume emission appears in the vicinity of the substrate, while the primary plume emission seems to be pushed backward towards the target. Due to geometrical restrictions, the measurements were taken at an angle of incidence of $45^{\circ}$, as compared to the normal incidence geometry used for thin-film deposition. The spot size will increase from the circular shape $(b / a$ ratio $\sim 1)$ to an elliptical one with the $b / a$ ratio $\sim 1.4$. This will also lead to a narrower plume [13]. This means that under the normal incidence geometry, the plume emission would appear more broadened. We believe that the plume dynamics and processes that occur at the substrate are similar.

In order to identify the fast species in the plume, the energy distribution of $\mathrm{Au}$ and $\mathrm{Cu}$ ions was measured with an ion-energy analyzer (see Supplemental Material [17]). The measurements on the ion-energy distribution in vacuum reveal the presence of highly energetic species in the plume, with the energy tail of $\mathrm{Au}$ and $\mathrm{Cu}$ ions that extends well above $100 \mathrm{eV}$ (up to $200 \mathrm{eV}$ for $\mathrm{Cu}$ ions and $500 \mathrm{eV}$ for $\mathrm{Au}$ ions).

The composition of the films grown at different $\mathrm{Ne}$ background pressures ( $0.1 \mathrm{mbar}, 0.5 \mathrm{mbar}$, and $2 \mathrm{mbar})$ versus angle of deposition are shown in Fig. 4(a), left-hand plots. The deposits are Cu-poor/Au-rich and the degree of $\mathrm{Cu}$ nonstoichiometry decreases with increasing pressure. At 2 mbar, the composition of the deposits is relatively similar over a wide range of angles varying from $-90^{\circ}$ to $+90^{\circ}$. The corresponding thickness profiles of the deposits are shown in Fig. 4(b), right-hand plots. The profiles were fitted as explained above, and the fitting parameter, $k$, is shown in each figure. One can observe that $k$ gradually decreases as the $\mathrm{Ne}$ pressure increases from 0.1 mbar to $2 \mathrm{mbar}$, suggesting that the plume broadens as a consequence of the interaction with the background gas.

Finally, the effect of the heavy background gas (Xe) on the angle-dependent composition of the $\mathrm{AuCu}$ deposits is shown in Fig. 5. A clear broadening of the thickness and composition profiles is observed as compared to vacuum. Nevertheless, the deviation from stoichiometry is comparable with the deposits 

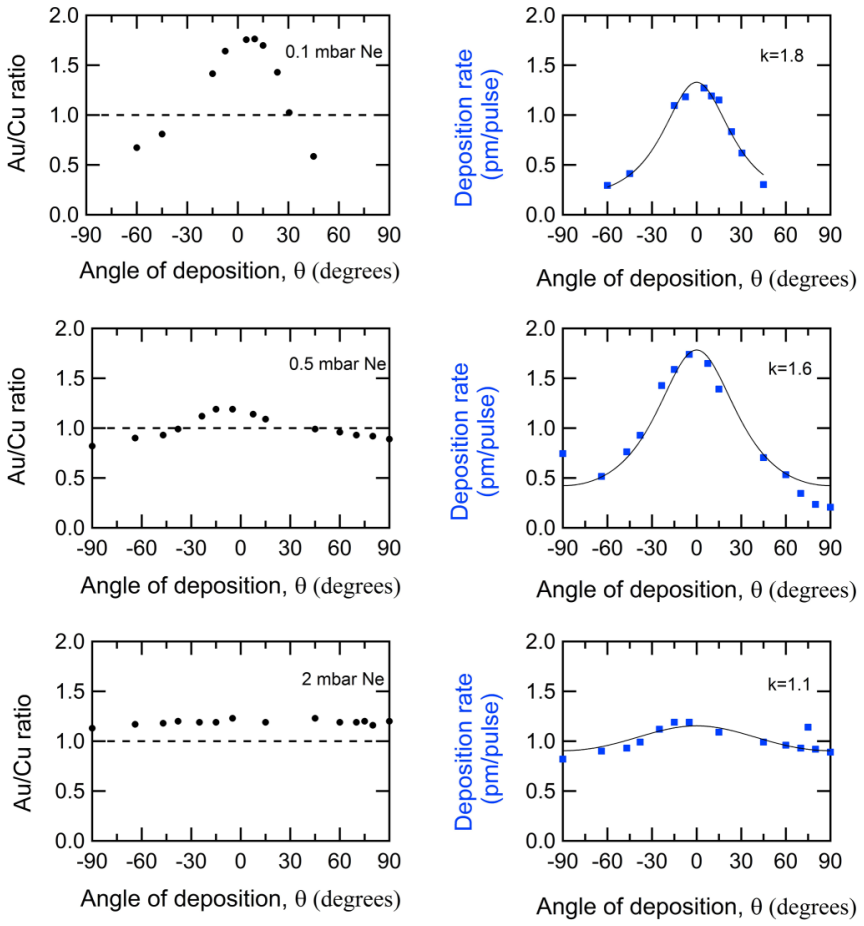

FIG. 4. (a) Left-hand plots: Ratio of elements $(\mathrm{Au} / \mathrm{Cu})$ versus angle of deposition in $\mathrm{Ne}$ at $0.1 \mathrm{mbar}, 0.5 \mathrm{mbar}$, and $2 \mathrm{mbar}$ at a laser fluence of $5 \mathrm{Jcm}^{-2}$. The dashed line represents the composition of the target. (b) Right-hand plots: Angular thickness profiles fitted using Anisimov's model and the fitting parameter $(k)$, which describes the broadening of the deposition yield.

in vacuum. The thickness profile could not be fitted using Anisimov's model.

\section{DISCUSSION}

The results presented here indicate that the composition of the $\mathrm{AuCu}$ films deposited in vacuum and in $\mathrm{Ne}$ - and Xe-background gases is off-stoichiometric, and the metal ratio in the films varies significantly as a function of laser fluence, pressure and mass of the background gas. Even though the depletion of the light elements in films deposited by PLD has been reported in the literature $[7,18,19]$, the degree of the nonstoichiometric transfer is significantly larger compared to findings on other materials [18-21]. The mechanisms involved in the noncongruent deposition in PLD are (i) preferential evaporation of the volatile components from the target, (ii) preferential scattering of the light-mass species in the plume, (iii) atom/ion reflection on the substrate surface, and (iv) preferential (re)sputtering of the film by the energetic ablated species arriving at the substrate. Hereafter, we will discuss our experimental findings based on the processes mentioned above.

\section{A. Deposition in vacuum}

The noncongruent material transfer in vacuum is observed at both laser fluences; however, the effect is significantly enhanced at the high laser fluence of $5 \mathrm{Jcm}^{-2}$, where an enrichment in $\mathrm{Au}$ of $\sim 70 \%$ with respect to the composition of
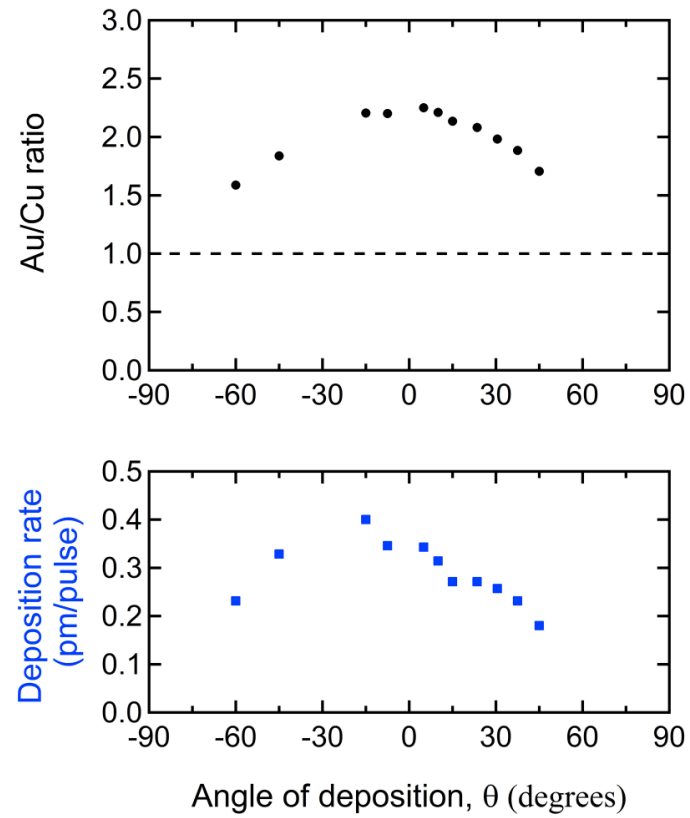

FIG. 5. Ratio of elements $(\mathrm{Au} / \mathrm{Cu})$ and thickness profile versus angle for deposition in Xe gas (0.01 mbar). The dashed line represents the composition of the target.

the target has been experimentally determined for films grown at angles near normal incidence. Films deposited at large angles are stoichiometric. The spectroscopic plume imaging reveals a strong distortion of the plume emission followed by the appearance of a secondary emission at the substrate position [Fig. 3(b)]. Since this effect is mostly pronounced at a laser fluence of $5 \mathrm{Jcm}^{-2}$ this indicates that microscopic-scale processes, as will be discussed below, play an important role on the $\mathrm{Cu}$ loss in the films.

The sputtering of a single-component target is generally well understood [22], and self-sputtering occurs when the kinetic energy of the ablated species is at least one order of magnitude higher than the cohesive energy of the target atoms. Mass-resolved ion-energy distributions of the ions reveal a high-energy tail for the ions, which extends up to $200 \mathrm{eV}$ for $\mathrm{Cu}$ and $500 \mathrm{eV}$ for $\mathrm{Au}$, as shown in the Supplemental Material [17]. This implies that resputtering/ion mixing can be efficient above a threshold energy of $\sim 35 \mathrm{eV}$, which corresponds to the sputtering threshold for pure $\mathrm{Cu}$ (Table I). For two-component or multicomponent targets, such as the $\mathrm{AuCu}$ alloy, it has been experimentally determined that (i) the cohesive energy of atoms in an alloy is smaller than that in a pure element, and (ii) the atoms with the highest cohesive energy remain those with the highest value in an alloy [23]. The ratio between the sputtering yield of component 1 of mass $M_{1}$, concentration $c_{1}$, and cohesive energy $U_{01}$ and a component 2 of mass $M_{2}$, concentration $c_{2}$, and cohesive energy $U_{02}$ is given by:

$$
\frac{Y_{1}}{Y_{2}}=\frac{c_{1}}{c_{2}}\left(\frac{M_{2}}{M_{1}}\right)^{2 m}\left(\frac{U_{02}}{U_{01}}\right)^{1-2 m},
$$

where $m$ is an exponent from the interaction potential [23]. Since $m$ is typically around $0.05-0.1$, and the cohesive energies of $\mathrm{Au}$ and $\mathrm{Cu}$ are similar, the ratio $\frac{Y_{1}}{Y_{2}}$ depends only weakly on the cohesive energy and even less on the mass of the 
elemental constituents. Equation (2) predicts a tendency (of $25 \%$ magnitude) for lighter elements to be preferentially sputtered with respect with the heavy masses. Note that this estimation is purely theoretical since the cohesive energy of the atoms will be smaller than that in a crystalline alloy because of the higher number of defects in the film formed during bombardment of energetic ions. Any significant change in the cohesive energy of $\mathrm{Au}$ and $\mathrm{Cu}$ can thus modify the estimate above. The preferential sputtering of $\mathrm{Cu}$ with respect to $\mathrm{Au}$ can partly explain the Au-rich deposits at small angles without being the sole effect.

The apparent recoil of the plume front indicates that the atom/ion reflection at the substrate can play a role as well, similarly with the findings by Gonzalo et al. [11]. The backscattered ions can interact with electrons resulting in the formation of neutrals via electron-capture and subsequent electron-induced excitation of the neutrals [24]. The interaction of the backscattered ions with the incoming ions reaching the substrate can induce preferential scattering of the light $\mathrm{Cu}$ atoms in the plume. Thus, the nonstoichiometric material transfer at high fluence can arise from resputtering, ion backscattering, as well as differential scattering of the light species in the plume.

The spatial and angular distribution of the plume in vacuum is forward peaked along the target surface normal $[25,26]$, meaning that the resputtering, as well as backscattering efficiency decline progressively at larger angles. Consequently, a quasistoichiometric deposition is achieved at large angles.

\section{B. Deposition in neon and xenon background gases}

The plume dynamics in background gas has been studied intensively [6,27-29]. In particular, a splitting of the plume into an energetic component traveling at near vacuum speed and a component slowed by the ambient gas has been observed [30]. The plume splitting has been studied for ablation of metals, such as Ag [31], as well as of complex compounds such as $\mathrm{LiMn}_{2} \mathrm{O}_{4}$ [6], $\mathrm{LaAlO}_{3}$ [32], etc. The background gas appears to act as a regulator of highly energetic ablated species. While the dynamical behavior of the plume has been widely studied, a qualitative estimate of the material deposited on the substrate as a function of the mass and pressure of the background gas has been poorly investigated.

The data presented here indicate that the angular profile of the deposition yield broadens with increasing background pressure [Fig. 4(a)], while the Cu nonstoichiometry in the films is reduced, suggesting that the Ne background gas reduces the kinetic energy of the ablated species. In particular, the angular distribution of the deposits at 2 mbar Ne is very broad, such that the dependence on the angle of deposition is smeared out. This indicates that the $\mathrm{Au}$ and $\mathrm{Cu}$ atoms/ions are thermalized upon multiple collisions with the background gas and reach the substrate through diffusion. In the diffusionlike regime, the atoms/ions arriving at the substrate might have suffered many collisions with the background gas and reduced their initial kinetic energy (up to $500 \mathrm{eV}$ in case of $\mathrm{Au}$ ions) to values below the sputtering threshold (i.e., $\sim 35 \mathrm{eV}$ for pure $\mathrm{Cu})$. Interestingly, the deposits collected at low angles are $\mathrm{Cu}$ poor, and the composition merges into $\mathrm{Cu}$-rich films at large angles, predominantly at a background pressure of 0.1 and 0.5 mbar Ne. This interesting trend resembles a differential scattering of $\mathrm{Cu}$ in plume with respect to $\mathrm{Au}$; the larger the pressure during deposition, the broader the angular scattering of the $\mathrm{Cu}$ atoms in the plume. At a pressure of $2 \mathrm{mbar} \mathrm{Ne}$, the films are still $\mathrm{Cu}$ poor $(\sim 12 \%$ with respect to the composition of the target) and the angular composition of the deposits is similar over the entire hemisphere. A similar behavior was observed for $\mathrm{La}_{0.6} \mathrm{Ca}_{0.4} \mathrm{MnO}_{3}$ in Ar gas [19].

In the presence of Xe gas, the transfer is noncongruent and comparable in magnitude with the deposits in vacuum. All peaks broaden considerably over their distributions in vacuum. However, the mechanism responsible for the loss of $\mathrm{Cu}$ might be different.

To understand the scattering between the ablated species and the background gas, one can consider the simple case of one-dimensional elastic collisions, in which all the velocities lie along the positive $x$ axis. Let us consider $m_{a}$ and $m_{g}$ as the masses of the plume species and background gas, respectively. We define the corresponding $x$ velocities of the ablated species and gas before the collision $v_{a x}$ and $v_{g x}$, and those after collision $v_{a x}^{\prime}$ and $v_{g x}^{\prime}$. In case of head-on collisions, the velocity of the plume species after collision are given by:

$$
v_{a x}^{\prime}=\frac{m_{a}-m_{g}}{m_{a}+m_{g}} v_{a x}+\frac{2 m_{g}}{m_{a}+m_{g}} v_{g x},
$$

If the mass of the ablated plume species is equal with the mass of the gas $\left(m_{a}=m_{g}\right)$ and $v_{g x}$ is small and positive, the particles exchange only velocities upon collision.

\section{Collisions in Ne gas:}

For $\mathrm{Cu}$ in $\mathrm{Ne}\left(m_{a}>m_{g}\right)$ :

$$
v_{a x}^{\prime}=0.51 v_{a x}+0.46 v_{g x}, \quad v_{a x}^{\prime}>0 .
$$

For $\mathrm{Au}$ in $\mathrm{Ne}\left(m_{a}>m_{g}\right)$

$$
v_{a x}^{\prime}=0.81 v_{a x}+1.81 v_{g x}, \quad v_{a x}^{\prime}>0,
$$

Eqs. (4) and (5) are valid when $v_{g x}$ is small and positive.

\section{Collisions in Xe gas:}

For $\mathrm{Cu}$ in $\mathrm{Xe}$ gas $\left(m_{a}<m_{g}\right)$, Eq. (3) gives:

$$
\begin{aligned}
v_{a x}^{\prime}= & -0.34 v_{a x}+1.34 v_{g x}, \\
& v_{a x}^{\prime}>0 \text { if } v_{g x}>0.25 v_{a x}, \quad \text { otherwise } v_{a x}^{\prime}<0 .
\end{aligned}
$$

For $\mathrm{Au}$ in Xe gas $\left(m_{a}>m_{g}\right)$ :

$$
v_{a x}^{\prime}=-0.19 v_{a x}+0.79 v_{g x}, \quad v_{a x}^{\prime}>0,
$$

Eqs. (6) and (7) are satisfied when $v_{a x}$ and $v_{g x}$ are positive.

For $\mathrm{Au} / \mathrm{Cu}$ in $\mathrm{Ne}$, the velocity of the species after collision $v_{a x}^{\prime}>0$, meaning that the plume species are never scattered in the backward direction. For $\mathrm{Au} / \mathrm{Cu}$ in $\mathrm{Xe}$, Eq. (6) implies that there will be backscattering of the $\mathrm{Cu}$ atoms by the $\mathrm{Xe}$ atoms until the gas particles have reached $25 \%$ of the velocity of the plume species. The heavy Au species will not experience backscattering effects.

We will now consider a situation when background gas particles are stationary but the collisions are not head-on. We 

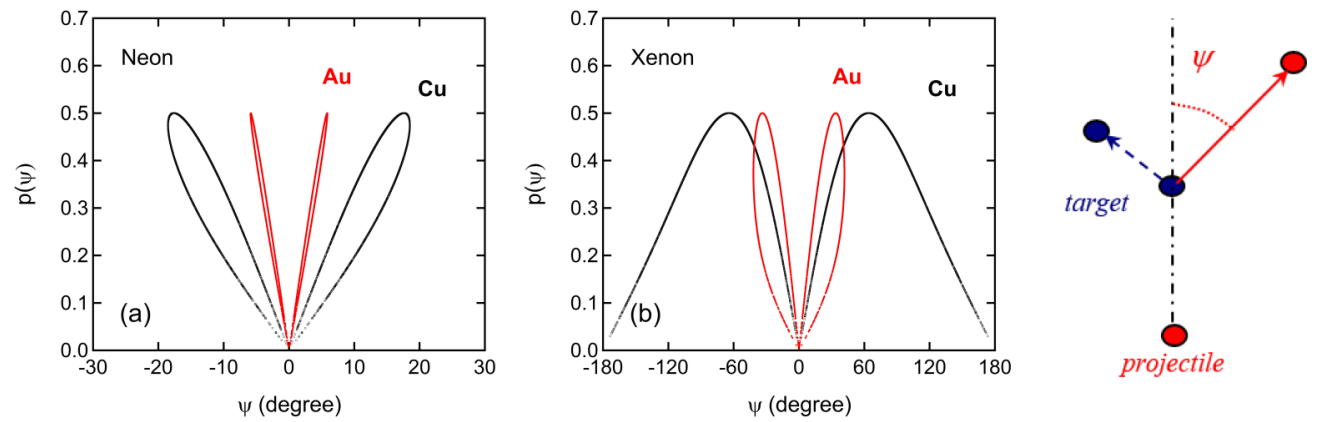

FIG. 6. Collisional scattering probability $p(\psi)$ versus scattering angle $\psi$ for the collisional dynamics of $\mathrm{Au}$ and $\mathrm{Cu}$ atoms with (a) $\mathrm{Ne}$ and (b) Xe. Note the different $X$ scale between the plots. The schematic of a collision and the scattering angle $\psi$ is shown to the right of the panels. Values of the scattering angle $\psi$ larger than $90^{\circ}$ denotes backscattering.

define $p(\psi)$ as the scattering probability between the plume species and the background gas atoms and $\psi$ the scattering angle between the direction of $\mathrm{Au}$ and $\mathrm{Cu}$ before and after an elastic collision [33]. The scattering probability for a single scattering event between the projectile $(\mathrm{Au}, \mathrm{Cu})$ and target $(\mathrm{Ne}, \mathrm{Xe})$ atoms, as calculated by Monte Carlo simulation is shown in Figs. 6(a) and 6(b). The $p(\psi)$ function is symmetric with respect to $\psi=0$. The scattering in a binary collision is isotropic in the center of mass (COM) reference frame of the system, but anisotropic in the laboratory reference frame. It is important to mention that the scattering angle $\psi$ depends on the $m_{g} / m_{a}$ ratio. When the ratio is larger than 1, i.e., $\mathrm{Au}$ and $\mathrm{Cu}$ in $\mathrm{Ne}$, the same value of $\psi$ can result from a couple of values of the scattering angle $\psi$ in the COM frame. The same applies for $\mathrm{Au}$ in $\mathrm{Xe}$. Hence, two values of $p(\psi)$ up to a maximum scattering angle $\psi_{\max }$ of $6(\mathrm{Cu}$ in $\mathrm{Ne}), 19$ (Au in $\mathrm{Ne}$ ), and 42 degrees ( $\mathrm{Au}$ in $\mathrm{Xe}$ ) are obtained. For $\mathrm{Cu}$ in $\mathrm{Xe}$, only one value $\psi$ exists for any scattering angle in the COM reference frame. In the presence of $\mathrm{Ne}$, an average scattering angle for $\mathrm{Au}$ and $\mathrm{Cu}$ of $\sim 5$ and $\sim 15$ degrees, respectively, was determined. This implies that $\mathrm{Cu}$ species will be more broadly scattered by the ambient Xe gas than Au. Moreover, the scattering angle $\psi$ varying from -180 to 180 degrees and $|\psi|>90$ degrees indicates scattering of the plume species in the backward direction. Figure 6 reveals the Au atoms are not backscattered while the $\mathrm{Cu}$ atoms experience a backscattering (of $\sim 25 \%$ ). Furthermore, these simple calculations indicate that $\mathrm{Cu}$ is more broadly scattered by the $\mathrm{Xe}$ atoms as compared to $\mathrm{Au}$, which allows us to conclude that ablation in the heavy Xe gas can lead to preferential scattering of the light massspecies in the plume, which in turn can explain the $\mathrm{Cu}$ loss for the deposits in Xe.

\section{CONCLUSION}

In summary, the elemental distribution of $\mathrm{Au}$ and $\mathrm{Cu}$ in the films deposited in vacuum and in the background gases of $\mathrm{Ne}$ and Xe was investigated over a wide range of angles of deposition. Films deposited near normal incidence have an elemental ratio $\mathrm{Au}: \mathrm{Cu}$ of 1.2 at a laser fluence of $2.5 \mathrm{Jcm}^{-2}$ and 2.5 at a laser fluence of $5 \mathrm{Jcm}^{-2}$. At large angles, the material transfer in vacuum is always stoichiometric. Time-resolved images of the plume emission in vacuum reveal a distortion of the plume and the appearance of a secondary emission at the substrate, distinctly observed at the high laser fluence of $5 \mathrm{Jcm}^{-2}$. The loss of $\mathrm{Cu}$ in the $\mathrm{AuCu}$ films was attributed to two distinct processes, i.e., preferential resputtering of $\mathrm{Cu}$ and differential scattering of the lightweight $\mathrm{Cu}$ atoms in the plume. In the presence of a background gas, a more complex behavior is observed as a function of the atomic mass and pressure of the gas. Films deposited in the light Ne gas have an elemental ratio of $\mathrm{Au} / \mathrm{Cu}$ which broadens significantly with increasing pressure, resulting in films with an even composition at all angles at a pressure of $2 \mathrm{mbar} \mathrm{Ne}$. In Xe, the degree of the nonstoichiometry is comparable with that in vacuum. Calculations show that $\mathrm{Cu}$ atoms are more broadly scattered as compared to Au atoms upon collisions with Xe; furthermore, the calculations indicate a backscattering probability of $\mathrm{Cu}$ in $\mathrm{Xe}$ of $\sim 25 \%$. Both effects can be responsible for the loss of $\mathrm{Cu}$ in the films deposited in the heavy Xe gas.

\section{ACKNOWLEDGMENTS}

The authors would like to acknowledge Nini Pryds and Anders Christian Wulff for their help in the preparation of the $\mathrm{AuCu}$ target by arc melting.
[1] S. Das, K. Sen, I. Marozau, M. A. Uribe-Laverde, N. Biskup, M. Varela, Y. Khaydukov, O. Soltwedel, T. Keller, M. Döbeli, C. W. Schneider, and C. Bernhard, Phys. Rev. B 89, 094511 (2014).

[2] S. Amoruso, R. Bruzzese, N. Spinelli, R. Velotta, M. Vitiello, and X. Wang, Phys. Rev. B 67, 224503 (2003).

[3] A. Cazzaniga, A. Crovetto, C. Yan et al., Sol Energ Mat Sol C 166, 91 (2017).
[4] T. Ohnishi, M. Lippmaa, T. Yamamoto, S. Meguro, and H. Koinuma, Appl. Phys. Lett. 87, 241919 (2005).

[5] S. Wicklein, A. Sambri, S. Amoruso, X. Wang, R. Bruzzese, A. Koehl, and R. Dittmann, Appl. Phys. Lett. 101, 131601 (2012).

[6] S. Canulescu, E. L. Papadopoulou, D. Anglos, T. Lippert, C. W. Schneider, and A. Wokaun, J. Appl. Phys. 105, 063107 (2009). 
[7] T. C. Droubay, L. Qiao, T. C. Kaspar, M. H. Engelhard, V. Shutthanandan, and S. A. Chambers, Appl. Phys. Lett. 97, 124105 (2010).

[8] F. Simmen, T. Lippert, P. Novak, B. Neuenschwander, M. Doebeli, M. Mallepell, and A. Wokaun, Appl. Phys. A: Mater. Sci. Process. 93, 711 (2008).

[9] E. Breckenfeld, N. Bronn, J. Karthik, A. R. Damodaran, S. Lee, N. Mason, and L. W. Martin, Phys. Rev. Lett. 110, 196804 (2013).

[10] A. Ojeda-G-P, C. W. Schneider, M. Dobeli, T. Lippert, and A. Wokaun, J. Appl. Phys. 121, 135306 (2017).

[11] J. Gonzalo, J. Siegel, A. Perea, D. Puerto, V. Resta, M. Galvan-Sosa, and C. N. Afonso, Phys. Rev. B 76, 035435 (2007).

[12] C. Klamt, A. Dittrich, B. Jaquet, C. Eberl, F. Doering, and H. Krebs, Appl. Phys. A: Mater. Sci. Process. 122, 701 (2016).

[13] B. Toftmann, J. Schou, and J. G. Lunney, Phys. Rev. B 67, 104101 (2003).

[14] A. Ojeda-G-P, C. W. Schneider, T. Lippert, and A. Wokaun, J. Appl. Phys. 120, 225301 (2016).

[15] S. Anisimov, B. Lukyanchuk, and A. Luches, Appl. Surf. Sci. 96-98, 24 (1996).

[16] S. I. Anisimov, D. Bauerle, and B. S. Lukyanchuk, Phys. Rev. B 48, 12076 (1993).

[17] See Supplemental Material at http://link.aps.org/supplemental/ 10.1103/PhysRevMaterials.1.073402 for more information on the ion energy distribution in vacuum.

[18] M. C. Foote, B. B. Jones, B. D. Hunt, J. B. Barner, R. P. Vasquez, and L. J. Bajuk, Physica C 201, 176 (1992).
[19] A. Ojeda-G-P, C. W. Schneider, M. Doebeli, T. Lippert, and A. Wokaun, Appl. Surf. Sci. 336, 150 (2015).

[20] W. S. Choi, Z. Marton, S. Y. Jang, S. J. Moon, B. C. Jeon, J. H. Shin, S. S. A. Seo, T. W. Noh, K. Myung-Whun, H. N. Lee, and Y. S. Lee, J. Phys. D: Appl. Phys. 42, 165401 (2009).

[21] C. Arnold and M. Aziz, Appl. Phys. A: Mater. Sci. Process. 69, S23 (1999).

[22] J. Schou, Appl. Surf. Sci. 255, 5191 (2009).

[23] P. Sigmund, Nucl. Instrum. Methods Phys. Res. Sect. B-Beam Interact. Mater. Atoms 82, 192 (1993).

[24] E. Thomas, Prog. Surf. Sci. 10, 383 (1980).

[25] T. N. Hansen, J. Schou, and J. G. Lunney, Appl. Phys. Lett. 72, 1829 (1998).

[26] B. Doggett and J. G. Lunney, J. Appl. Phys. 109, 093304 (2011).

[27] T. Donnelly, J. G. Lunney, S. Amoruso, R. Bruzzese, X. Wang, and X. Ni, J. Appl. Phys. 108, 043309 (2010).

[28] S. Harilal, C. Bindhu, M. Tillack, F. Najmabadi, and A. Gaeris, J. Appl. Phys. 93, 2380 (2003).

[29] J. Gonzalo, C. Afonso, and I. Madariaga, J. Appl. Phys. 81, 951 (1997).

[30] S. Amoruso, A. Sambri, and X. Wang, Appl. Surf. Sci. 253, 7696 (2007).

[31] S. Amoruso, B. Toftmann, and J. Schou, Phys Rev E. 69, 056403 , (2004).

[32] A. Sambri, A. Khare, S. Mirabella, E. Di Gennaro, A. Safeen, F. Di Capua, L. Campajola, U. S. di Uccio, S. Amoruso, and F. M. Granozio, J. Appl. Phys. 120, 225306 (2016).

[33] R. F. Wood, K. R. Chen, J. N. Leboeuf, A. A. Puretzky, and D. B. Geohegan, Phys. Rev. Lett. 79, 1571 (1997). 